\title{
Modern trends in clinical anatomy teaching
}

\section{Editorial}

Recent rapid advancements in information and communication technology have changed the paradigm of medical education. Computer networks, web based learning, and portable network devices are becoming a fundamental component of the learning environment in medical education, having an impact on design, and re-shaping the medical curriculum. A vast wealth of information is available at the student's fingertips; automated learning systems, interactive programs are all available to be used in class and for independent study. These new communication and information technologies are being used to enhance the teaching and learning environment. They have the capacity to facilitate student learning and problem solving, allowing for a better integration of the didactic approach with clinical practice; making the transition between learning in a classroom and clinical practice smoother. Formal lectures are still the backbone of medical education and are the predominant form of learning, as they allow imparting large amounts of factual information in an organized fashion to a large student audience. Current teaching technologies allow making lectures more interesting using techniques such as drawing on smart boards, video fragments, "moving" images, and others. Using clinical scenarios and question discussion during the lecture time makes students to be active participants of the learning process especially with the aid of feedback systems such as the clicker technology. Modern students are primarily active learners, and pure lecture courses may be increasingly out of touch with how students engage in their world. Of course, relatively 'passive' lectures will only get you so far, and individual teaching by apprenticeship supported by self-directed study remain necessary for learning specialized and high level skills.

Instead of classical textbooks, students now use e-books on laptops or tablet computers. In core courses, such as first year anatomy many medical schools don't use dissection anymore; professionally dissected plastinated specimens and virtual 3D body systems are used instead. Class schedule, professors' presentations, and exams, are available online now. Courses are predominantly delivered online via systems such as the Learning Management System (LMS) or a Virtual Learning Environment (VLE). Effective course management systems handle and control the delivery of learning courses provide online exams and manage teacher-students communication. The LMS tracks the learners' activities, progress, compliance with course assignments facilitate peer to peer as well as professor to student communication and much more. Many universities provide students with lecture broadcast, allowing for access to lectures anywhere and anytime in the 'virtual classroom'. Information technologies are particularly important in the current medical curriculum, where the shorter course of anatomy, too often forces the teaching of all anatomical details of the subject away from its clinical fulfillment. But it is imperative that medical students think like future physicians from the very beginning. This is where advancements in IT are imperative for bridging the gap between pure didactic lectures and interactive learning thus closer resembling clinical scenarios by allowing for on the feet problem solving. Some examples of these developments include the following. Simulation or imitation using technology of real life circumstances
Volume 2 Issue I - 2016

\author{
Volodymyr Mavrych \\ Department of Anatomy, St. Matthew's University, UK
}

Correspondence: Volodymyr Mavrych, Department of

Anatomy, St. Matthew's University, UK,

Email vmavrych@stmatthews.edu

Received: January II, 2016 | Published: January 18, 2016

such as anatomic regions or clinical procedures is particularly useful. An example of simulation is a Virtual dissection table. It provides realistic visualization of $3 \mathrm{D}$ anatomical details of virtual cadaver. A full body gross anatomy model was rendered from CT scan data. The Table can also open any data from CT, MRI, and ultra-sound scanners. Unlike cadavers, the students can redo and undo the dissection again and again. Virtually slicing, layering, and segmenting the anatomy can customize different body views providing operation procedures in a natural and intuitive manner.

Traditionally used tools such as plain radiographs are commonly used for trauma, bony fractures and joint dislocations along with chest and $\mathrm{x}$-rays of the skull.Computed tomography (CT) and magnetic resonance imaging (MRI), both are intensively utilized in most of the anatomy courses. Ultrasonography is widely used for all aspects of medicine and ultrasound images should be present in Gross Anatomy labs along with radiographs, CTs and MRIs. Since ultrasound is a non-invasive imaging method, a machine may be located directly at a lab to provide students with a necessary experience and skills. Developments in ultrasound technology (probes size and frequency range) allow scanning the different areas of the body. Endoscopy (gastroduodenoscopy, colonoscopy, and bronchoscopy) is a very useful nonsurgical procedure used to examine cavities of empty organs. Endoscopic images of the esophagus, stomach, duodenum, large intestine and bifurcation of the trachea may be used on the radiographic station to reinforce students understanding of different clinical applications, which are discussed during the lab session. For those who must learn or relearn human anatomy without access to cadavers, there's a critical need for learning tools that present images of the real human body in three dimensions. There are two different approaches in the presentation of three-dimensional images: stereoscopic vision and rotation. Stereoscopic vision is used for everyday depth perception, and it's the basis for all 3D images and movies. These visual aids all require special equipment (3D glasses and screens). There is another way - rotation of the object. Object rotation is what we do without thinking, every time we're curious to learn something about a solid object. It gives our seeing brain a succession of images that it synthesizes to create a three dimensional mental image. Once we have that three-dimensional image, any two-dimensional image of the object that we see later makes threedimensional sense. Many applications on portable handheld electronic 
devices maximize access to resources from any environment. They are routinely used by students for medical questions, interactive games, and allow the students to get used to using electronic applications that will later become useful in patient management, and treatment decisions. Many medical apps focus on anatomy and physiology and other subjects, some address medical problem solving, diagnosis, and treatment. Furthermore social media such as Facebook, Twitter and other applications allow students to connect and schedule tutorial sessions, group study sessions and facilitate the sharing of information and peer-to-peer learning. Assessment is an integral part of any course. It's an evaluation of students' knowledge, a final product of the teaching. Currently most of the quizzes and exams are computer based and integrated in the Learning Management System. Starting from weekly quizzes and finishing with NBME subject shelf exams, all assessments may be done on the base of students' laptops or school computer class desktops in a way of working stations. LMS also provides survey capabilities. Surveys may be used for both pre- and post-course assessment. They allow the instructor to assess students' knowledge of topics to assist in course design or to facilitate different aspects of his teaching. As a post-course tool, surveys allow to evaluate student progress or to assess the instructor performance.

\section{Acknowledgements}

None.

\section{Conflict of interest}

Author declares that there is no conflict of interest. 